\title{
Carte électronique générique à base de compensateurs avance-retard pour les travaux pratiques d'automatique continue
}

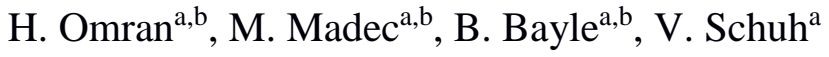 \\ ${ }^{a}$ Télécom Physique Strasbourg, Université de Strasbourg, Strasbourg, France \\ ${ }^{\mathrm{b}}$ Laboratoire ICube, Université de Strasbourg / CNRS, Strasbourg, France \\ Contact email : morgan.madec@unistra.fr
}

\begin{abstract}
Dans le cadre des travaux pratiques d'automatique en première année à Télécom Physique Strasbourg, nous avons conçu une carte électronique unique permettant la réalisation d'une large gamme de correcteurs pour l'asservissement continu sur des systèmes divers : moteur, système de chauffage, lévitation magnétique. La carte est constituée de trois circuits d'entrée permettant l'implémentation d'un filtre du premier ordre, de quatre modules correcteurs constitués chacun d'un compensateur à avance-retard de phase, d'un additionneur et d'un soustracteur, ainsi que de trois sorties. Cette maquette permet aux étudiants de réaliser facilement différents asservissements en interconnectant les blocs entre eux et en ajustant les valeurs de composants passifs (résistances et condensateurs) de sorte à obtenir les fonctions de transfert calculées en préparation. L'utilisation de la maquette est illustrée dans cet article sur l'un des exercices de TP : l'asservissement en position d'une bille magnétique.
\end{abstract}

\section{Introduction}

L'automatique continue est enseignée à Télécom Physique Strasbourg en première année sous la forme d'un module de 64 heures incluant 12 heures de TP sous MATLAB et 12 heures de TP sur maquette (1). Les étudiants y découvrent les notions de systèmes et asservissements, de représentation d'un système sous la forme de fonctions de transfert et de représentations d'état, les réponses impulsionnelles, indicielles et fréquentielles des systèmes, les outils mathématiques permettant l'analyse de la stabilité des systèmes (diagramme de Bode, diagramme des phases, lieu d'Evans ...) ainsi que la commande des systèmes continus et la notion de correcteurs (PI, PID, avance et retard de phase, retour d'état, ...)

La partie pratique sur maquette inclut trois TP d'asservissement sur trois maquettes permettant d'illustrer la diversité des procédés : un système instable (bille en lévitation), la commande d'un système lent à retard (chaufferette) et la commande d'un moteur à courant continu en position et en vitesse. Jusqu'à présent, les correcteurs associés à ces TP étaient réalisés à l'aide de pupitres de commande analogique de type OPALE 1 .

Nous avons développé récemment une carte électronique dédiée à ces TP. L'objectif était bien évidemment de remplacer les pupitres vieillissants, mais également de donner une vision plus concrète du correcteur (le pupitre étant une boîte noire sur laquelle on règle des boutons) et de renforcer le lien entre les enseignements d'automatique continue et les enseignements d'électronique analogique. Ce papier décrit cette carte électronique générique et le type de correcteur qu'elle permet de réaliser (section II). Son utilisation 
dans le cadre des TP est décrite sur un exemple d'asservissement de position dans une maquette de lévitation magnétique (section III).

\section{Description de la carte}

La carte électronique réalisée est représentée sur la figure 1. Elle est composée de trois circuits d'entrée, quatre compensateurs avance-retard, quatre additionneurs, quatre soustracteurs et trois circuits de sorties. Les blocs peuvent être interconnectés entre eux par des câbles externes. Le circuit est alimenté avec une alimentation externe +12/-12 V.

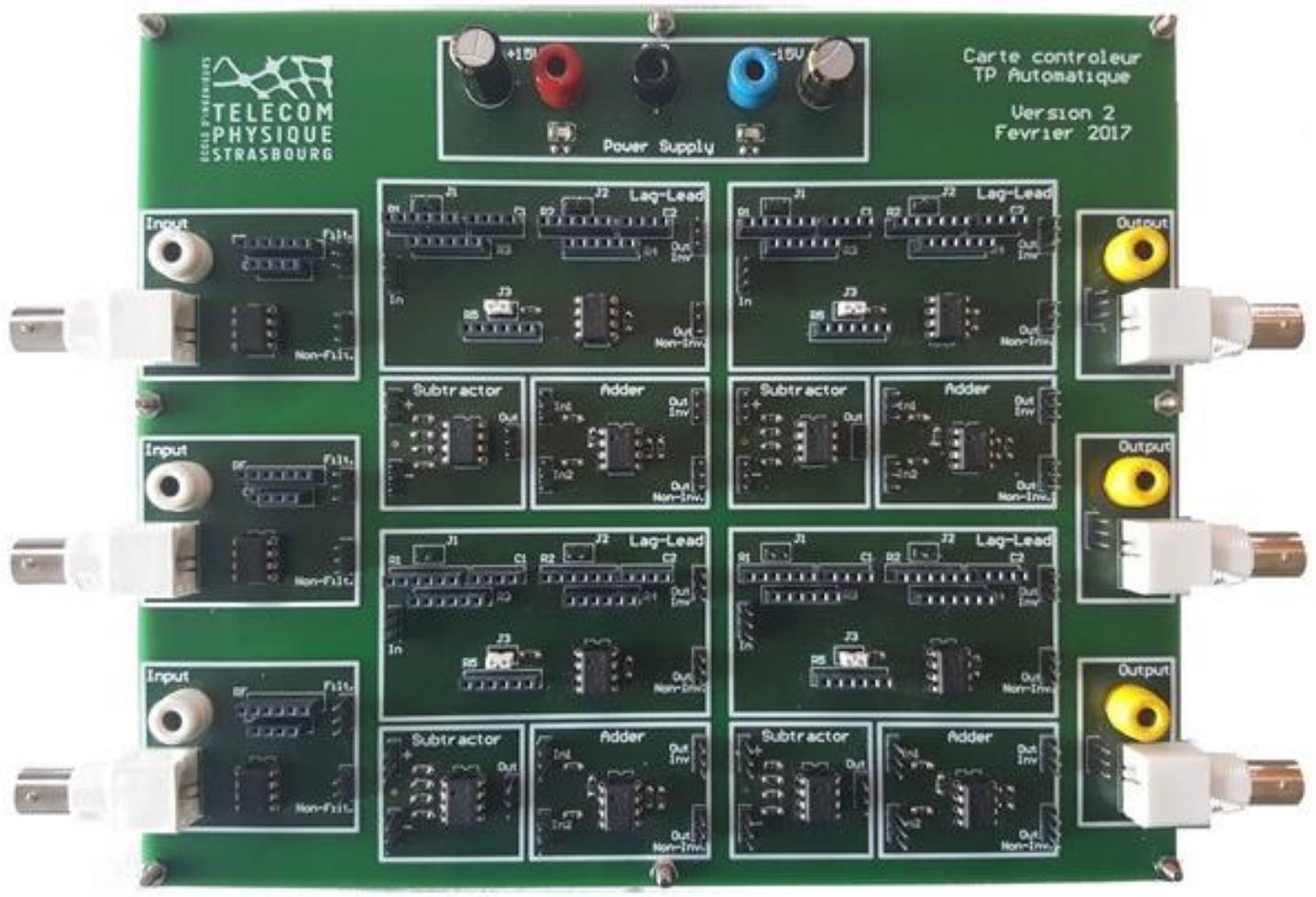

Fig.1. Photo de la carte électronique réalisée pour les TP d'automatique.

Le circuit d'entrée est constitué d'un connecteur, d'un filtre R-C de premier ordre ajustable et d'un amplificateur opérationnel. Il est possible de choisir, en sortie de chaque bloc, entre le signal brut et le signal filtré.

Le compensateur avance-retard, qui constitue l'essentiel de la carte, est décrit sur la Figure 2. Il est constitué de deux étages : le compensateur lui-même et un inverseur à gain fixe (égal à -1 en connectant $L_{3}$ ) ou ajustable (via $\underline{R}_{5}$ ). L'utilisateur peut choisir en sortie du bloc entre le signal issu directement du compensateur et celui issu de l'inverseur. La fonction de transfert réalisée par ce bloc est ajustée par le choix des valeurs de 5 résistances, de 2 condensateurs et le positionnement de 3 cavaliers.

\section{Simple gain}

Cette fonction est obtenue en n'utilisant que les résistances $R_{3}$ et $R_{4}$ et en utilisant l'inverseur de gain -1 si on souhaite un gain positif. 


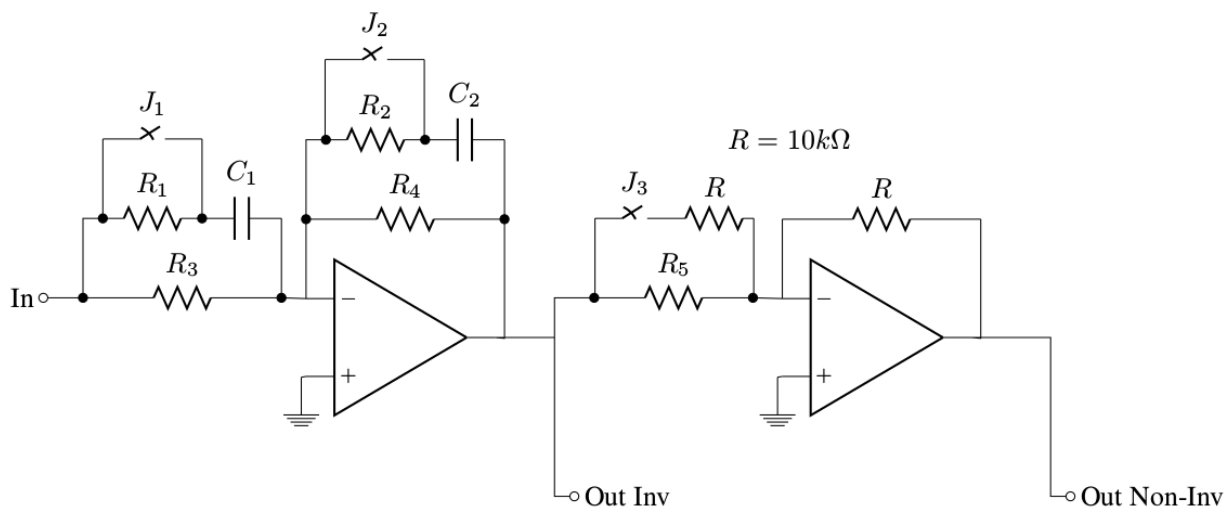

Fig.2. Schéma du compensateur avance-retard.

\section{$\underline{\text { Intégrateur }}$}

Cette fonction est obtenue en utilisant le condensateur $C_{2}$ et la résistance $R_{3}$ et en connectant le cavalier $J_{2}$. La résistance $R_{4}$ peut être également utilisée pour limiter la bande passante de l'intégrateur et ainsi réduire l'instabilité à basse fréquence en obtenant un filtre de premier ordre. La fonction de transfert réalisée par le circuit est:

$$
H(s)=\frac{-R_{4}}{R_{3}} \cdot \frac{1}{1+R_{4} \cdot C_{2} \cdot s}
$$

Le rapport $-R_{4} / R_{3}$ permet de régler le gain du filtre tandis que le produit $R_{4} \cdot C_{2}$ permet de fixer la pulsation à partir de laquelle le circuit opère en intégrateur.

\section{Dérivateur filtré}

Afin d'éviter les problèmes d'instabilité et d'amplification du bruit à haute-fréquence, on réalise un dérivateur avec filtrage du premier ordre. La fonction de transfert est obtenue en utilisant les résistances $R_{1}$ et $R_{4}$ et le condensateur $C_{1}$ :

$$
H(s)=-R_{4} \cdot C_{1} \frac{s}{1+R_{1} \cdot C_{1} \cdot s}
$$

Le produit $-R_{4} \cdot C_{1}$ sert à régler le gain du dérivateur tandis que le produit $R_{1} \cdot C_{1}$ permet de fixer la pulsation jusqu'à laquelle le dérivateur doit opérer.

\section{Proportionnelle-Intégrale}

Cette fonction est obtenue en utilisant les résistances $R_{3}$ et $R_{2}$ et le condensateur $C_{2}$. La fonction de transfert réalisée par le circuit est :

$$
H(s)=\frac{-R_{2}}{R_{3}}-\frac{1}{R_{3} \cdot C_{2} \cdot S}
$$

Le gain proportionnel est $-R_{2} / R_{3}$ ainsi que le gain intégrateur est $-1 /\left(R_{3} . C_{2}\right)$. Une autre stratégie pour réaliser un correcteur PI à l'aide de cette carte consiste à configurer un module en intégrateur, un autre en simple gain puis à sommer les signaux issus de ces deux modules.

\section{Correcteur à avance-retard de phase}

Cette fonction est obtenue en utilisant les résistances $R_{3}$ et $R_{4}$ et les condensateurs $C_{1}$ et $C_{2}$ et en connectant les cavaliers $J_{1}$ et $J_{2}$. La fonction de transfert est alors : 


$$
H(s)=\frac{-R_{4}}{R_{3}} \cdot \frac{1+R_{3} \cdot C_{1} \cdot s}{1+R_{4} \cdot C_{2} \cdot s}
$$

Pour le retard de phase, le gain $K$ est fixé par le rapport $-R_{4} / R_{3}$. Pour le retard de phase, la constante de temps $T$ et le paramètre $b$ (voir Tableau 1) sont donnés respectivement par le produit $R_{3} \cdot C_{1}$ et le rapport $\left(R_{4} \cdot C_{2}\right) /\left(R_{3} . C_{1}\right)$. Pour l'avance de phase, la constante de temps $T$ et le paramètre $a$ sont donnés respectivement par le produit $R_{4} \cdot C_{2}$ et par le rapport $\left(R_{3} . C_{1}\right) /\left(R_{4} \cdot C_{2}\right)$.

\section{Correcteur proportionnelle-intégrale-dérivée}

Le correcteur proportionnelle-intégrale-dérivée n'est autre que la somme d'un correcteur PI et d'un correcteur dérivateur filtré. Avec la maquette cette réalisation est possible en utilisant un compensateur réglé en PI, un compensateur réglé en Dérivateur filtré et enfin un sommateur. La fonction de transfert réalisée par le circuit est alors :

$$
H(s)=H_{P I}(s)+H_{D}(s)=\frac{-R_{2}}{R_{3}}-\frac{1}{R_{3} \cdot C_{2} \cdot s}-\frac{R_{4} \cdot C_{1} \cdot s}{1+R_{1} \cdot C_{1} \cdot s}
$$

Pour le bloc PI, le gain proportionnel est donné par $-R_{2} / R_{3}$ et le gain intégrateur par $-1 /\left(R_{3} \cdot C_{2}\right)$. Pour le bloc dérivateur filtré, le gain est donné par $-R_{4} \cdot C_{1}$. Enfin, $1 /\left(R_{1} \cdot C_{1}\right)$ correspond à la pulsation jusqu'à laquelle le dérivateur doit opérer.

$\underline{\text { Bilan }}$

Les correcteurs réalisables sont résumés dans le Tableau 1. Pour chaque correcteur présenté, d'autres configurations sont possibles en combinant plusieurs blocs compensateurs et les additionneurs et soustracteurs de la carte. Ces configurations alternatives peuvent avoir un intérêt dans des cas extrêmes pour permettre un meilleur ajustement des paramètres de correcteurs. D'autres correcteurs, réalisables en utilisant les modules avance-retard, sont illustrés en (2).

TABLEAU I. Résumé des correcteurs réalisables

\begin{tabular}{ccl}
\hline Fonction & \multicolumn{1}{c}{ Équation caractéristique } & \multicolumn{1}{c}{ Réglages } \\
\hline Proportionnel & $K$ & $K=-R_{4} / R_{3}$ \\
Intégrateur (HF) & $\frac{K_{I}}{1+T \cdot s}$ & $K_{I}=-R_{4} / R_{3}$ et $T=R_{4} \cdot C_{2}$ \\
$\begin{array}{c}\text { Dérivateur filtré } \\
\text { (BF) }\end{array}$ & $\frac{K_{D} \cdot s}{1+T_{D} \cdot s}$ & $K_{D}=-R_{4} \cdot C_{1}$ et $T_{D}=C_{1} \cdot R_{1}$ \\
PI & $K_{P}+\frac{K_{I}}{S}$ & $K_{P}=-R_{2} / R_{3}$ et $K_{I}=-1 /\left(R_{3} \cdot C_{2}\right)$ \\
Retard de phase & $K \cdot \frac{1+T \cdot s}{1+b \cdot T \cdot s}$ & $K=-R_{4} / R_{3}, T=R_{3} \cdot C_{1}$ et $b=\left(R_{4} \cdot C_{2}\right) /\left(R_{3} \cdot C_{1}\right)$ \\
Avance de phase & $K \cdot \frac{1+a \cdot T \cdot s}{1+T \cdot s}$ & $K=-R_{4} / R_{3}, T=R_{4} \cdot C_{2}$ et $a=\left(R_{3} \cdot C_{1}\right) /\left(R_{4} \cdot C_{2}\right)$ \\
PID = PI + & $K_{P}+\frac{K_{I}}{S}+\frac{K_{D} s}{1+T_{D} \cdot s}$ & $K_{P}=-R_{2} / R_{3}$ et $K_{I}=-1 /\left(R_{3} \cdot C_{2}\right)$ de PI. $K_{D}=-R_{4} \cdot$ \\
Dérivateur filtré & $C_{D}=R_{1} \cdot C_{1}$ de dérivateur \\
\hline
\end{tabular}

\section{III.Application : asservissement en position d'une bille magnétique}

Le principe de la lévitation magnétique est un sujet de recherche actuel en automatique (3). Il est utilisé dans plusieurs technologies comme les trains à suspension magnétique et 
les paliers magnétiques actifs. Pour étudier ce principe, dans le cadre des travaux pratiques en automatique, on considère le dispositif MAGLEV de la société Quanser (4). Il s'agit d'un système, non linéaire, à un degré de liberté composé d'une bille en acier qui peut être maintenue en lévitation sous l'action d'un électro-aimant (l'actionneur).

Le courant dans l'électro-aimant, dont dépend la force magnétique exercée, est mesuré à l'aide d'un capteur résistif. Par ailleurs, la position de la bille est mesurée à l'aide d'un photo-Darlington. Ce système a donc une entrée (la tension appliquée à l'électro-aimant) et deux sorties (les mesures du courant de l'électro-aimant et de la position de la bille).

Afin de piloter le système en boucle fermée, les étudiants trouvent d'abord un modèle linéaire autour d'un point de fonctionnement. Ensuite, ils réalisent un asservissement de type cascade, c'est-à-dire formé par deux boucles imbriquées (Figure. 3). La première boucle (interne) permet d'asservir le courant dans la bobine ; la seconde permet d'asservir la position de la bille. Enfin, la mesure de la tension fournie par le capteur de position fait apparaître un bruit, donc une cellule de filtrage est utilisée pour filtrer ce bruit.

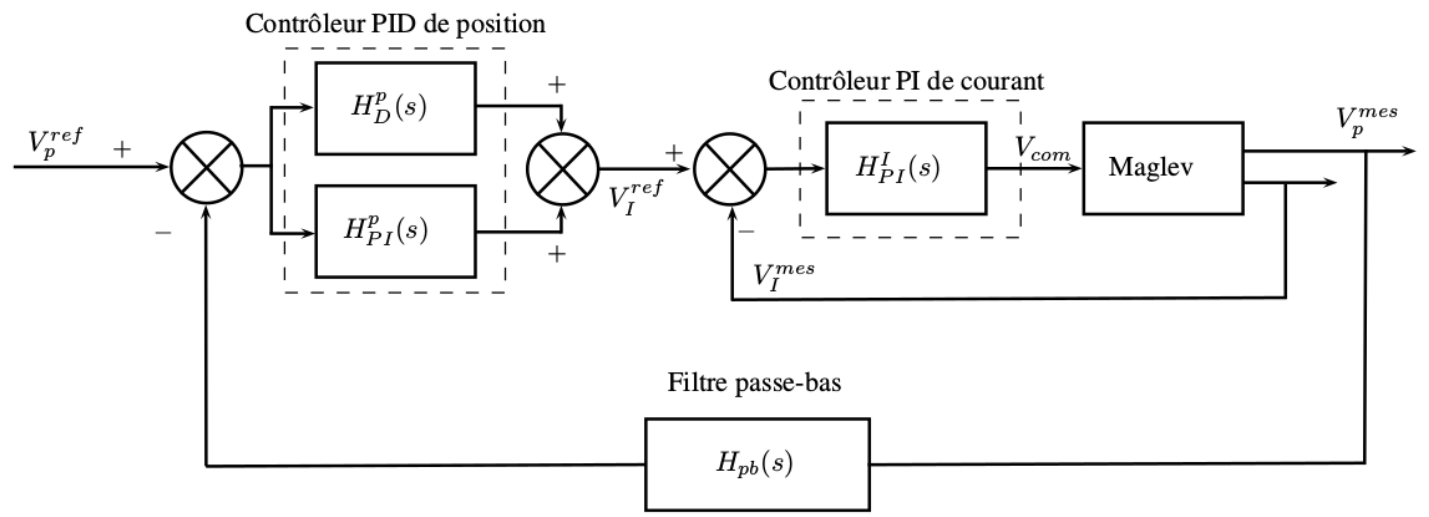

Fig.3. Principe de l'asservissement cascade du MAGLEV constitué de deux boucles : un PID pour l'asservissement de la position et un PI pour l'asservissement du courant.

Pendant la préparation du TP, les élèves synthétisent les fonctions de transfert de la boucle d'asservissement (nous ne détaillons pas leurs calculs). Nous présentons, dans la suite, ces fonctions de transfert ainsi que la réalisation de la boucle de commande en utilisant notre carte. La fonction de transfert du filtre passe-bas est :

$$
H_{P B}(s)=\frac{1}{1+1.5810^{-4} . s}
$$

Le correcteur de type PI pour la boucle de courant est :

$$
H_{P I}^{I}(s)=19.02+\frac{660}{s}
$$

Enfin, le correcteur de type PID pour la boucle de position est :

$$
H_{P I D}^{P}(s)=H_{P I}^{P}(s)+H_{D}^{P}(s)=0.614+\frac{9.8 \cdot 10^{-3}}{s}+0.654 \cdot s+9.81 \cdot 10^{-4} \cdot s
$$

Pendant la séance de TP, les élèves proposent un montage AOP permettant de réaliser les correcteurs décrits par les équations (6), (7) et (8) comme illustré en figure 4. 


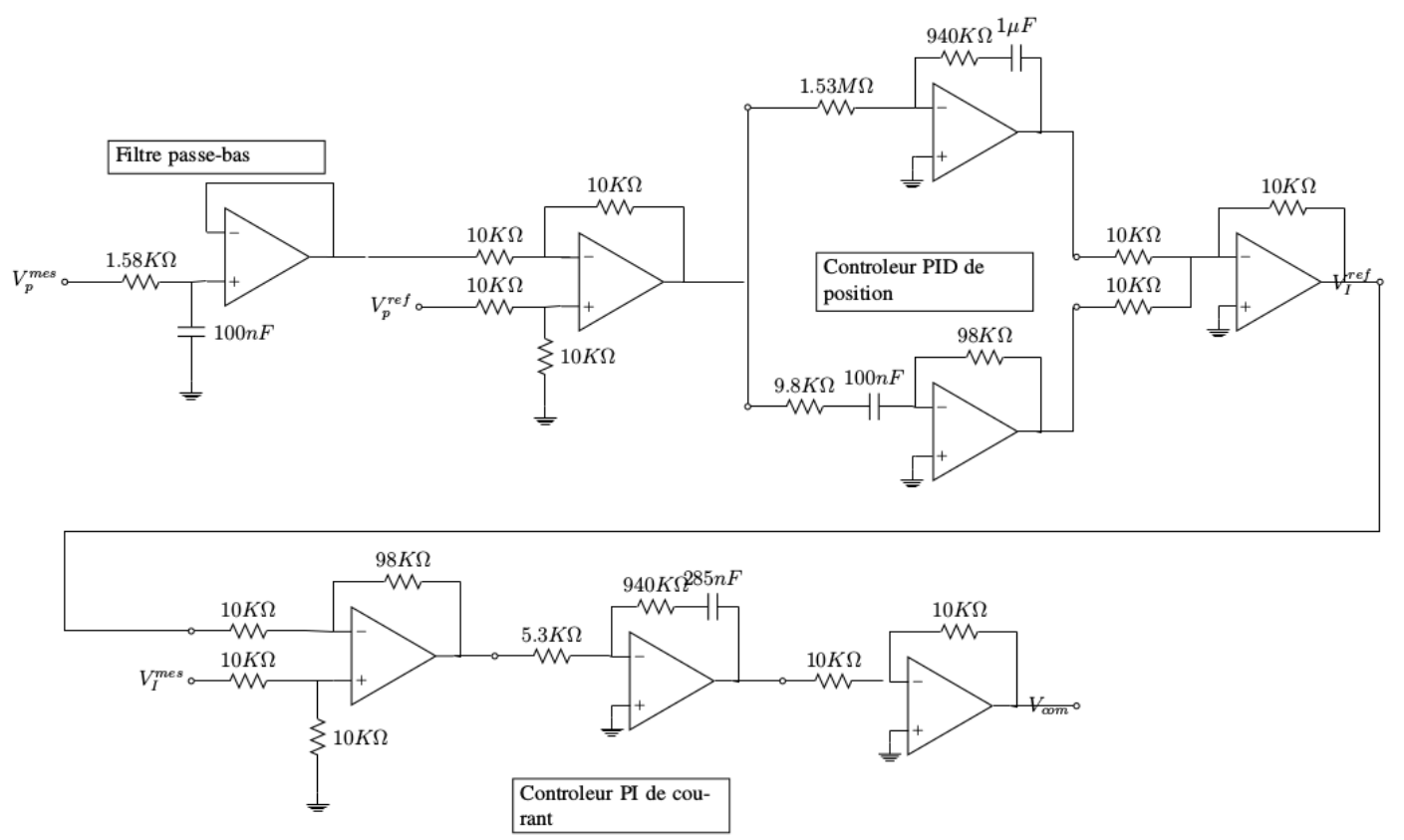

Fig.4. Schéma électrique de l'asservissement cascade du MAGLEV constitué de deux boucles : un PID pour l'asservissement de la position et un PI pour l'asservissement du courant.

Ensuite, les élèves doivent réaliser la boucle de commande complète à l'aide de la carte générique, comme illustrée en figure 5. Sur la figure, le correcteur en courant est décrit en bleu tandis que le correcteur en position est décrit en rouge. Un filtre passe-bas est implémenté dans le bloc d'entrée de la mesure de position. Le premier soustracteur calcule l'erreur de position. Celle-ci est envoyée en entrée de deux compensateurs, un configuré en PI et l'autre en dérivateur filtré. Les sorties de ces deux blocs arrivent ensuite sur un additionneur pour former un correcteur PID. La valeur en sortie du PID constitue la consigne en courant. Elle est soustraite à la mesure en courant pour calculer l'erreur en courant. Cette erreur arrive ensuite sur un $3^{\mathrm{e}}$ compensateur configuré en PI et dont la sortie sert de commande en courant.

Finalement, les élèves implémentent et testent le montage complet pour voir que la bille lévite et que $V_{p}$ suit $V_{p}^{\text {ref }}$ selon le cahier des charges. La figure 6 illustre le montage complet de l'asservissement du MAGLEV. La position de la bille est déterminée par un signal de consigne généré par un GBF. Malgré le fait que la boucle de commande contient plusieurs éléments en cascade, on peut remarquer que la carte permet d'avoir un montage simple ergonomique permettant un débogage efficace.

\section{Conclusion}

Nous avons constaté que ces cartes permettent aux étudiants d'apprendre d'une façon ludique en réalisant eux-mêmes les correcteurs et les filtres analogiques, ce qui permet de les sensibiliser aux aspects pratiques comme la saturation et l'amplification de bruit. À partir de cette année, ces cartes seront également utilisées au premier semestre dans le cadre des TP d'électronique analogique sur l'amplificateur opérationnel. Un nouveau TP permettra aux étudiants de se familiariser avec les cartes et d'effectuer des mesures qui ne sont pas traitées en TP d'automatique, telles que la mesure d'un diagramme de Bode ou encore l'étude des limitations de l'amplificateur opérationnel (effet de l'offset en intégrateur, par exemple). 


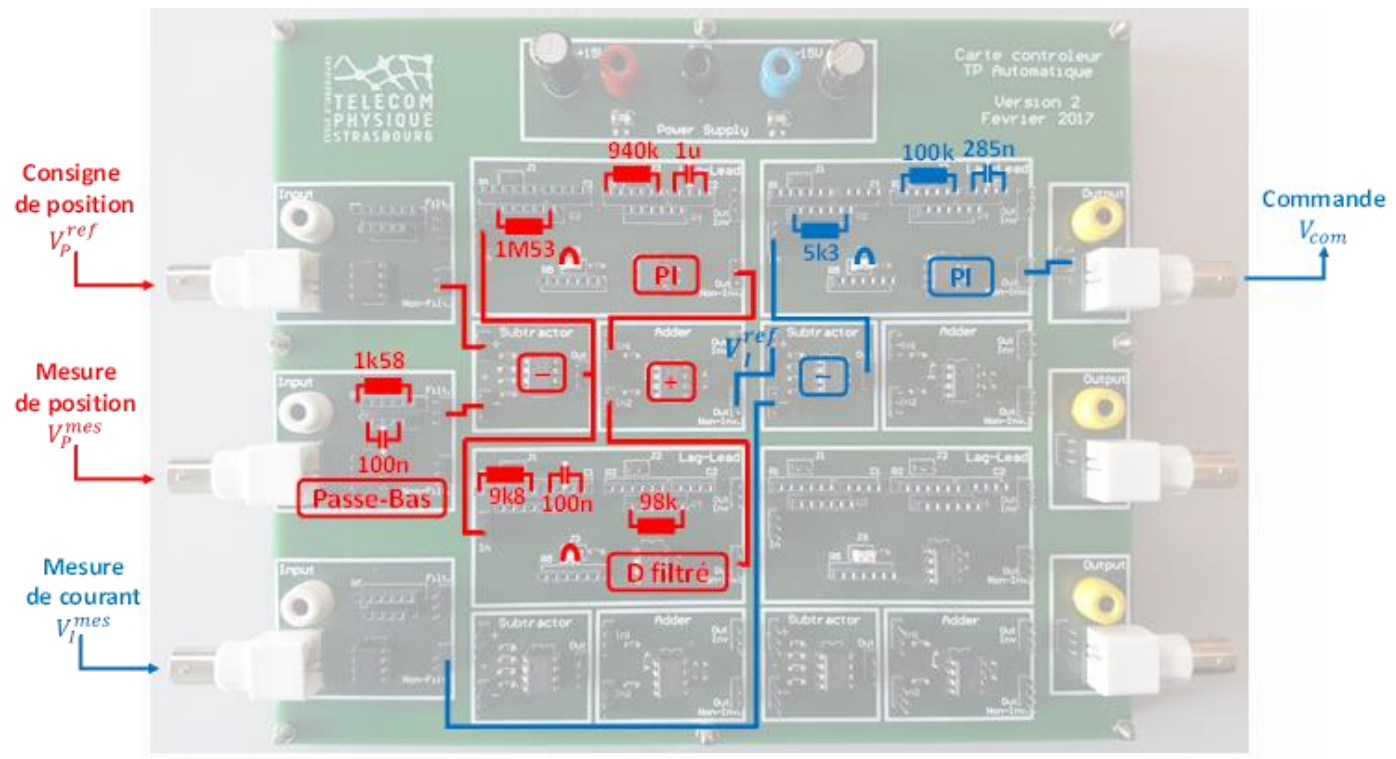

Fig.5. Implémentation des correcteurs décrits par les équations (6), (7) and (8) à l'aide de la carte électronique développée.

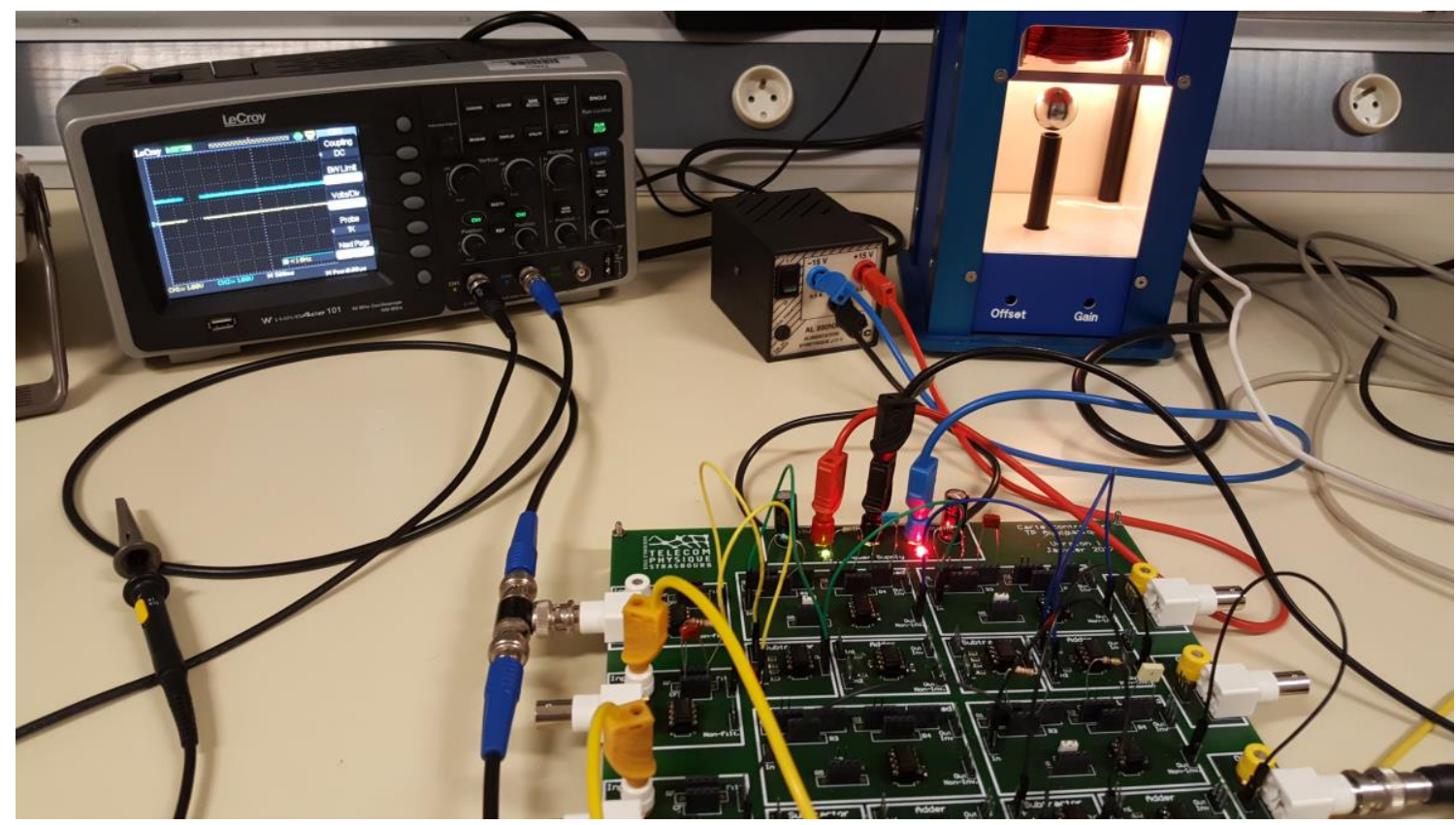

Fig.6. Photo de l'asservissement du MAGLEV en action. La bille est suspendue et sa position est déterminée par un signal de consigne généré par un GBF.

L'autre projet sur lequel nous travaillons également concerne la réalisation d'une maquette basée sur un Arduino, cette fois pour les TP de commande numérique, là encore pour renforcer le lien entre les enseignements d'électronique et d'automatique.

\section{Remerciements}

Les auteurs tiennent à remercier l'ensemble des collègues qui ont contribué d'une manière ou d'une autre à la mise en place de ces TP. La plupart des outils mis en œuvre dans ce TP ont été acquis grâce au soutien du GIP-CNFM (5) et du projet IDEFI FINMINA (6). 


\section{Références}

1. B. Bayle, Cours de systèmes et asservissements continus, Télécom Physique Strasbourg (2018).

2. K. Ogata, Modern Control Engineering, Prentice Hall, Fifth Edition (2009).

3. B. Yi , R. Ortega, W. Zhang, Relaxing the conditions for parameter estimation-based observers of nonlinear systems via signal injection, Systems \& Control Letters (2018).

4. Lévitation magnétique: maquette pédagogique pour enseigner la commande des systèmes nonlinéaires, Quanser, website: https://www.quanser.com/products/magnetic-levitation (Accès 2018).

5. GIP-CNFM: Groupement d'Intérêt Public - Coordination Nationale pour la formation en Microélectronique et en nanotechnologies. Website: $h t t p: / / w w w w . c n f m . f r$

6. IDEFI-FINMINA : Initiative d'Excellence - Formation Innovante en MIcroélectronique et Nanotechnologies, ANR-11-IDFI-0017. Website: http://www.cnfm.fr/VersionFrancaise/actualites/ FINMINA.htm 